\title{
Redundancy of noise elements and signals in visual detection of letters*
}

\author{
W. K. ESTES \\ The Rockefeller University, New York, New York 10021
}

\begin{abstract}
Forced-choice detection was studied in relation to heterogeneity of noise letters and multiple signal letters within tachistoscopic displays. Curves plotting both probability and latency of correct detections vs number of redundant signals at different display sizes exhibit significant interactions with heterogeneity of the noise background, but do not differ as a function of similarity among redundant signals. Application of interactive and independent channels models indicated that noise redundancy operates to a major extent through effects of signal-noise confusability at the decision level, but may also involve perceptual interaction at the level of feature detectors.
\end{abstract}

There is abundant evidence that identification of a target, or signal, letter in a background of noise letters depends strongly upon both the redundancy of the noise elements (Estes, 1972; McIntyre, Fox, \& Neale, 1970; McLaughlin, Masterson, \& Herrmann, 1972) and the multiplicity of signals (Estes, 1972; Kinchla, 1974; Wolford, Wessel, \& Estes, 1968). These variables have been the subject of considerable attention in connection with current models for processing of tachistoscopic displays, but in neither case is the nature of the functional relationship entirely clear.

Redundancy of noise elements might be expected to affect signal detection at either the perceptual or the decision level. Taking the extremes of redundancy, let us compare the task of detecting a given signal letter in a background of identical noise letters with detection of the same signal in a background of all-different noise letters. In a model proposed by the writer (Estes, 1972), inputs to feature detectors undergo inhibitory interactions. In the case of the heterogeneous noise background, more feature detectors will be activated than in the case of the homogeneous background; consequently, there will be more inhibition of the detectors involved in perception of the signal, yielding a lower detection probability and longer reaction time.

But the heterogeneous noise condition would also complicate the S's decision problem. On the average, the greater the number of different noise letters present in a display, the greater will be the likelihood that an imperfectly perceived noise letter will activate a sufficiently large subset of the feature detectors associated with a target letter to yield a false detection. This decision problem would be the primary locus of the predicted effect of noise redundancy in the models of Gardner (1973) and Shiffrin and Geisler (1973), but would be subsidiary to the hypothesized perceptual

*This research was supported in part by USPHS Grant GM16735 from the National Institute of General Medical Sciences. I wish to thank Edith Skaar for her substantial contribution to the data collection and processing and Richard M. Shiffrin and Ronald Kinchla for a number of valuable suggestions concerning the theoretical interpretations. interaction in the writer's formulation.

Though all of the relevant studies reported to date show that increasing heterogeneity of noise letters is detrimental to detection performance, none provides evidence bearing on the nature of the effect. The difficulty is that particular noise letters, when used in the homogeneous condition, differ significantly in their effects on performance (McIntyre, Fox, \& Neale, 1970); but in none of the studies reported has the selection of noise letters been fully equated between homogeneous and heterogeneous conditions. The first objective of the present study is to meet this requirement and thus to obtain a picture of the effect of different degrees of noise redundancy with effects of differential confusability of individual letters fully controlled.

It might be thought that if all of the noise letters used in an experiment occur with equal frequencies in homogeneous and heterogeneous displays, then there should be no effect of redundancy at the decision level. For the models of Estes (1972), Gardner (1973), and Shiffrin and Geisler (1973), all of which assume that inputs from the different noise letters of a display affect the decision process independently, this supposition is correct only if all noise letters are equally confusable with the signal letters. It can be shown that, if the noise letters differ in confusability, these models imply a facilitative effect of noise redundancy upon performance. The reason, in brief, is that when a relatively confusable noise letter occurs in the display with a given signal, any additional instances of the same noise letter in the same display will in a sense be wasted (see Appendix 1).

Turning to the effects of redundancy of the signal elements, we note that once again two factors which can be conceptually separated have been confounded in all studies reported. Multiple instances of a signal convey redundant information, but also they introduce physical similarity. I have assumed that signal redundancy exerts its effects primarily at the decision level, additional instances of a signal letter simply providing additional opportunities for identification (Estes, 1972), and it 
seems that a similar interpretation would follow from the models of Gardner (1973) and Shiffrin and Geisler (1973). But another possibility is that multiple instances of a signal might be mutually facilitating at the perceptual level as a consequence of their physical similarity. In Experiment II of the present study, an effort will be made to obtain a picture of the effects of signal redundancy independent of variation in physical similarity.

\section{EXPERIMENT I EFFECTS OF HETEROGENEITY OF NOISE CHARACTERS}

The primary objective of this experiment is to vary the redundancy of the noise letters within displays while equating over trials the frequencies with which particular letters occur in heterogeneous vs homogeneous displays. To this end, the population of noise elements is limited to four letters, each being selected equally often as the redundant noise element in homogeneous displays and each being included equally often as a constituent of heterogeneous displays. Display size and number of redundant signal elements per display will be included as additional experimental variables, combined factorially with noise redundancy. This design will provide evidence bearing on a model proposed by McLaughlin, Masterson, and Herrmann (1972), in which effects of redundancy are assumed to result from a perceptual process quite different in character from that of the interactive channels model.

\section{Method}

Apparatus. Character displays were presented on the screen of a Tektronix Type 564B storage oscilloscope under the control of a PDP-8/I computer. The $S$ sat at a table with a response box centered in front of him and faced a $50 \times 50 \mathrm{~cm}$ black panel $32 \mathrm{~cm}$ from the edge of the table. The screen of the oscilloscope was visible through a $10 \times 12 \mathrm{~cm}$ aperture in the panel. Displayed characters were $.75 \times .75 \mathrm{~cm}$ and an eight-letter array subtended ca $12 \mathrm{deg}$ visual angle. Displays were always centered on the screen. The two response buttons, inset into the response box, activated microswitches interfaced to the computer. Other details of the apparatus and displays were as described by Estes (1972).

Subjects. Twenty-four young adults were paid $\$ 2 / \mathrm{h}$ for their services. Most of them had had no previous experience as $S s$ in tachistoscopic experiments.

Design. The experimental variables were display size, number of signal elements in the display, and redundancy of the noise background. Two display sizes were used, four and eight elements. The signal letters were $D$ and $U$, each assigned to one response button and occurring equally often under all conditions. Each display contained 1, 2, 3, or 4 instances of the signal letter selected for that trial, the remaining spaces in the display being filled with noise letters. The noise letters used were $J, L, N$, and $P$. Under the high-redundancy condition, which we shall refer to as the constant background, some one of these noise letters was randomly selected for each trial and all of the positions in the display not occupied by the signals were filled with instances of this one noise letter (thus a four-element display containing two signals might be JDDJ). For the low-redundancy condition, which we shall refer to as the variable background, the noise letters required for any display were drawn randomly without replacement from the set comprising two instances of the letters $J, N, L$, and P.

The experimental program was organized by 48 -trial blocks, each block being assigned to a particular display size. Within a block, all four values for number of signal elements occurred equally of ten in a random sequence; within the set of 12 trials assigned to a particular number of signal elements, four displays were assigned to the variable background and eight to the constant background. The resultant 48-trial blocks were programmed on paper tape, and the order of presentation was counterbalanced over Ss.

Procedure. Except for a few minor changes, the procedure was as described in Estes (1972). At the beginning of the session, the $S$ read the instructions, which indicated that on each trial he was to fixate in the center of the screen, then view the display and press the response button indicating which of the two signal letters he believed was included in the display. Then he received a block of 48 practice trials (run under the nonconfusable background, Memory Set 4, Display Size 6 condition of Estes, 1972), data from which were not utilized, followed by the two experimental blocks.

Each trial began with a 2-sec exposure of a row of dot matrices, one filling the position of each letter that was to appear in the display, followed by a $100-\mathrm{msec}$ exposure of the letter display, after which the row of dot matrices reappeared and remained on the screen until the $S$ operated a response button. Then, following a $1-\mathrm{sec}$ blank interval, the premask for the next trial appeared, and so on.

\section{Results}

Proportions of correct detections are plotted as a function of number of signal elements in the upper panels of Fig. 1. An analysis of variance using as scores the proportions of correct detections by individual Ss under the various conditions, yields an estimated standard error of .025 for the points in the figure. The analysis shows the main effects of all three experimental variables to be significant; for constant vs variable background, $F=4.68, \mathrm{df}=1 / 23, \mathrm{p}<.05$; for display size, $F=17.22, \mathrm{df}=1 / 23, p<.01$; for number of signal elements, $F=49.29$, df $=3 / 69, p<.01$. Also, the interactions of Display Size by Number of Signals and of Background by Display Size by Number of Signals are significant, with $\mathrm{F}=5.83, \mathrm{df}=3 / 69, \mathrm{p}<.01$, and $\mathrm{F}=$ $2.91, \mathrm{df}=3 / 69, \mathrm{p}<.01$, respectively.

Mean reaction times for correct responses are plotted as a function of number of signals for each background and display size condition in the lower panels of Fig. 1. An analysis of variance yields an estimate of $.03 \mathrm{sec}$ for the standard error of the individual points in the figure. The analysis shows the effect of number of signals and the interaction of Background by Number of Signals to be significant $(F=22.66, \mathrm{df}=3 / 69, \mathrm{p}<.01$, and $\mathrm{F}=$ 3.73 , df $=3 / 69, p<.05$, respectively). As in the study of Estes (1972), correct reaction time does not vary appreciably with display size under either background condition. Latencies for errors are uniformly higher than those for correct responses, with an average in the neighborhood of $700 \mathrm{msec}$, and decrease with number of signals, but do not vary with background or display size. 
Fig 1. Proportions of correct detections (upper panels) and mean correct reaction times (lower panels) for Experiment I. The oarameter in each panel is variable vs constant noise background.

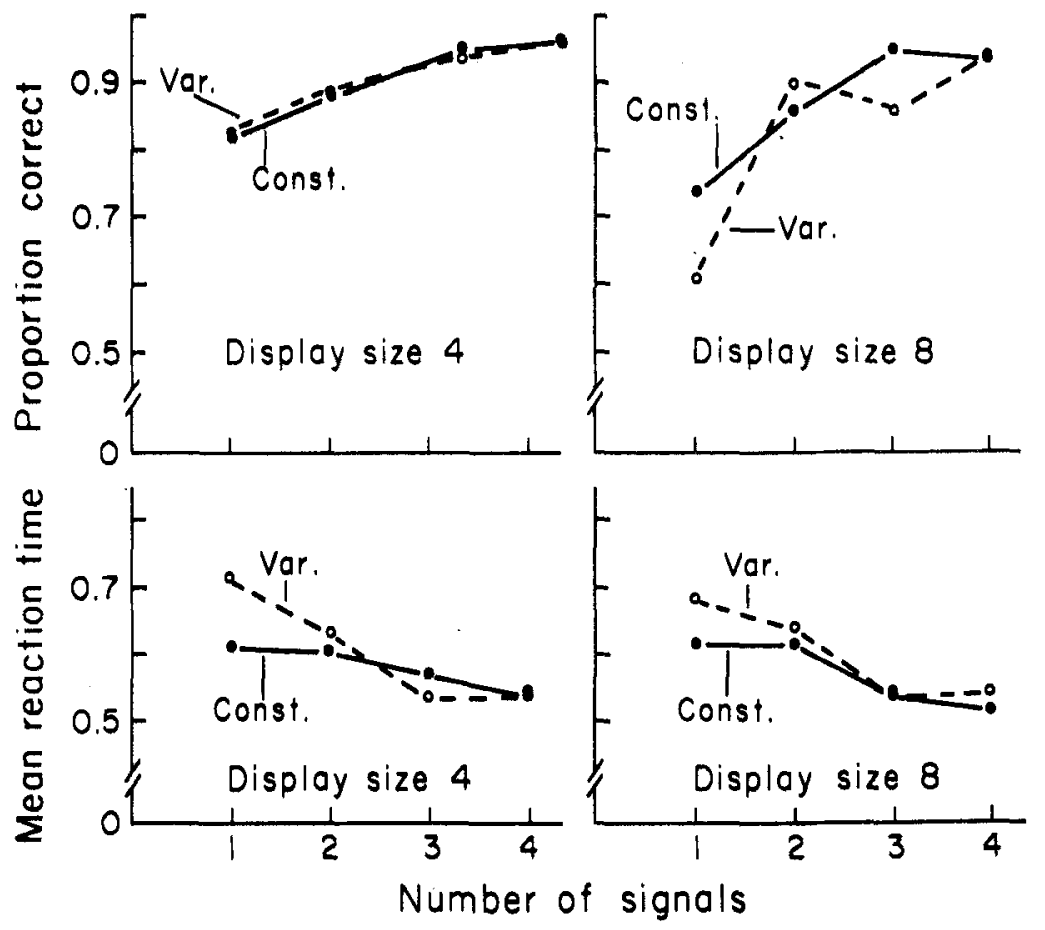

With regard to the primary objective of this experiment, it seems clear that, with other factors controlled, variation in redundancy of noise elements influences detection performance significantly. This result confirms the prediction of the interactive channels model (Appendix 2). However, on the reasoning illustrated in Appendix 1, the same result might be expected to arise from complications generated by heterogeneous noise elements at the decision level, provided that the individual noise elements differ among themselves in degree of confusability with the signals.

To check on this latter possibility, data for the homogeneous noise conditon were analyzed separately for the four noise letters, each of which was used on one-fourth of the trials in this condition. Error frequencies are $53,36,45$, and 37 for noise letters $J, L$, $\mathrm{N}$, and $\mathrm{P}$, respectively. The differences are doubtless insignificant, but with the small numbers of observations per $S$, no very sensitive test is possible. For correct reaction times, on the other hand, a relatively powerful test of differences can be obtained by computing a single mean reaction time for each $S$ on trials involving each noise letter and subjecting these scores to an analysis of variance. Mean reaction times on trials with noise letters $\mathrm{J}, \mathrm{L}, \mathrm{N}$, and $\mathrm{P}$, respectively, are $.66, .61, .63$, and $.59 \mathrm{sec}$, and the analysis of variance yields an $F$ of 1.46 with $3 / 69 \mathrm{df}$, where an $\mathrm{F}$ of 2.74 would be required for significance at the .05 level.

There remains the possibility that different noise letters might be differentially confusable with particular signal letters. To evaluate this possibility, we computed mean error frequencies for each combination of noise and signal letters on homogeneous noise trials. This analysis showed the noise letter $\mathrm{L}$ to yield equal error frequencies for the two signals, but $\mathbf{J}$ produced a preponderance $(68 \%)$ of errors when $\mathrm{D}$ was the signal, and $\mathrm{N}$ and $\mathrm{P}$ produced more errors $(69 \%$ and $65 \%$, respectively) when $U$ was the signal.

To provide a direct estimate of the influence of these differential signal-noise confusabilities upon error frequencies on heterogeneous noise trials, we computed the mean error frequencies separately for heterogeneous trials on which each of the two signal letters occurred in conjunction with different numbers of the more confusable noise letters ( $\mathrm{J}$ with $\mathrm{D}, \mathrm{N}$ or $\mathrm{P}$ with $\mathrm{U}$ ). For Display Size 4, mean errors per trial prove to be 2.0,3.6, and 3.5 on trials when 0,1 , or 2 confusable noise letters were present; for Display Size 8, mean errors are 0.7, $3.4,5.3$, and 6.6 for trials on which $0,1,2$, or 3 confusable noise letters were present. Clearly, a principal factor in the effect of noise redundancy on detection performance is the relation between heterogeneity of noise elements and number of opportunities for the occurrence of noise elements that are particularly . confusable with the signal present on a given trial.

\section{EXPERIMENT II REDUNDANCY OF MULTIPLE SIGNAL ELEMENTS}

Differential evidence bearing on the alternative interpretations of the multiple signal effect in terms of redundant information vs perceptual facilitation can be obtained by means of the following design. Two sets of signal elements are defined. In programming the experimental trials, one or the other of the two signal 
sets is chosen randomly on each trial and then one or more signal letters from the given set are included in the display along with a background of noise letters. Half of the trials are assigned to a duplicate signal condition, which in effect replicates the corresponding condition of Estes (1972); on these trials, 1, 2, 3, or 4 instances of some one signal element from one of the two signal sets will be present on any trial. The other half of the trials are assigned to a redundant signal condition which differs in that $1,2,3$, or 4 signals drawn from one of the signal sets, but not in general all identical, are included in the display on each trial. Thus, if $\mathrm{ABCD}$ constitutes a signal set, then on a trial which is programmed to include 3 signals under the duplicate condition three instances of the letter A might be included together with noise letters, whereas under the redundant condition an $\mathrm{A}, \mathrm{a} \mathrm{B}$, and a $\mathrm{C}$ might be included along with noise letters.

The two ways of varying redundancy of signals are precisely matched with respect to information value, but differ with respect to physical similarity or dissimilarity of the redundant signals present on any trial. On the basis of several current models (Estes, 1972; Gardner, 1973; Shiffrin \& Geisler, 1973), it must be predicted that if other factors are adequately controlled, the two conditions will yield similar results. Therefore, in the detailed design and analysis of the experiment, a primary focus of attention must be on the power of the test that is provided.

\section{Method}

The Ss, apparatus, and general procedures were all the same as in Experiment I. The new aspect of Experiment II was the utilization of two sets of signal letters in a manner which permitted two different ways of varying redundancy of multiple signals. The two sets were $S_{1}$, comprising the letters $A, B, C$, and $\mathrm{D}$ and assigned to the response button associated with the signal $D$ in Experiment $I$, and the set $S_{2}$, comprising the letters $S, T, U$, and $\mathrm{V}$ and assigned to the response button associated with the signal $U$ in Experiment $I$. Under the duplicate signal condition, $1,2,3$, or 4 instances of one of the signal letters from either $S_{1}$ or $S_{2}$ were included in the display on each trial. Under the redundant signal condition, $1,2,3$, or 4 different letters from either $S_{1}$ or $S_{2}$ were included in the display on each trial. For each of the two conditions, two 48-trial program tapes were prepared, one for Display Size 6 and one for Display Size 8. Within each 48-trial block, the signals of Sets $S_{1}$ and $S_{2}$ were represented equally of ten and the different numbers of signals, $1,2,3$, or 4 , occurred equally of ten on $S_{1}$ and $S_{2}$ trials. The signal letters selected for a trial were placed in random positions in the display, and the remaining positions were filled with noise letters, as in the variable background condition of Experiment I. Each $S$ received all four 48-trial blocks in a random sequence.

Each $S$ participated in Experiment II following Experiment I with a rest interval of about $5 \mathrm{~min}$ between the two. The only new instructions required for Experiment II were those needed to explain the assignment of signal let ters to response buttons.

\section{Results}

The overall results of this experiment will be immediately apparent from inspection of Fig. 2. In the upper panel, proportions of correct detections are plotted as a function of number of signal elements for each experimental condition. At each display size, the curves for redundant and duplicate signal conditions are as nearly superposed as could well be expected. An analysis of variance yields an estimate of .02 for the standard error of the individual points. The analysis shows the effect of display size and number of signal elements to be highly significant $(F=27.30, d f=1 / 23$, $\mathrm{p}<.01$, and $\mathrm{F}=61.25$, df $=3 / 69, \mathrm{p}<.01)$. By constrast, the $F$ for duplicate vs redundant signals is only .05 .

Mean reaction times for correct responses, shown in the lower panel of Fig. 2, reveal a similar picture. Again, the effects of display size and number of signal elements are significant $(\mathrm{F}=9.12, \mathrm{df}=1 / 23, \mathrm{p}<.01$, and $\mathrm{F}=$ $36.87, \mathrm{df}=3 / 69, \mathrm{p}<.01$ ), whereas the $\mathrm{F}$ for redundant vs duplicate signals is entirely nonsignificant, with a value of .02 . The analysis yields an estimate of .02 for the standard error of the plotted points in Fig. 2. There would seem to be no doubt but that the difference between redundant and duplicate multiple signals made no difference in detection performance and that the power of the experiment was sufficient to have revealed any appreciable difference had there been one.

It will be noted that the proportions of correct responses are lower and the latencies higher in this experiment than in Experiment I. This difference is to be expected in view of the fact that the memory set is larger in Experiment II. That is, in Experiment I, the S had only to keep representations of two possible signal letters in an active state in immediate memory in order to be prepared to detect occurrences of these in the displays, whereas in Experiment II the $S$ had to keep representations of eight possible signals in a similarly active state. As in my previous study (Estes, 1972), correct response latency increases with display size only at the larger value of size of memory set. The interpretation I have suggested for this relationship is that at larger display sizes there are fewer primary detections and therefore more instances in which S must attempt to arrive at a response by comparing the degraded inputs received from various positions in the display with elements of the memory sets. Increasing the size of the memory set from two to eight increases greatly the number of comparisons that must be made and thus should be expected to increase processing time.

\section{DISCUSSION}

With some additional controls, this study has confirmed the finding of previous investigations that both noise redundancy and signal redundancy facilitate the detection of a signal letter displayed for a brief exposure in a background of noise letters. Though it is difficult to make satisfactory comparisons across experiments conducted with somewhat different parameters, it appears that the effects of noise redundancy reported by McIntyre, Fox, and Neale (1970) and by McLaughlin, Masterson, and Herrmann 
Fig. 2. Proportions of correct detections times (lower panel) for Experiment II. The parameter in each panel is duplicate vs redundant multiple signals. (upper panels) and mean correct reaction
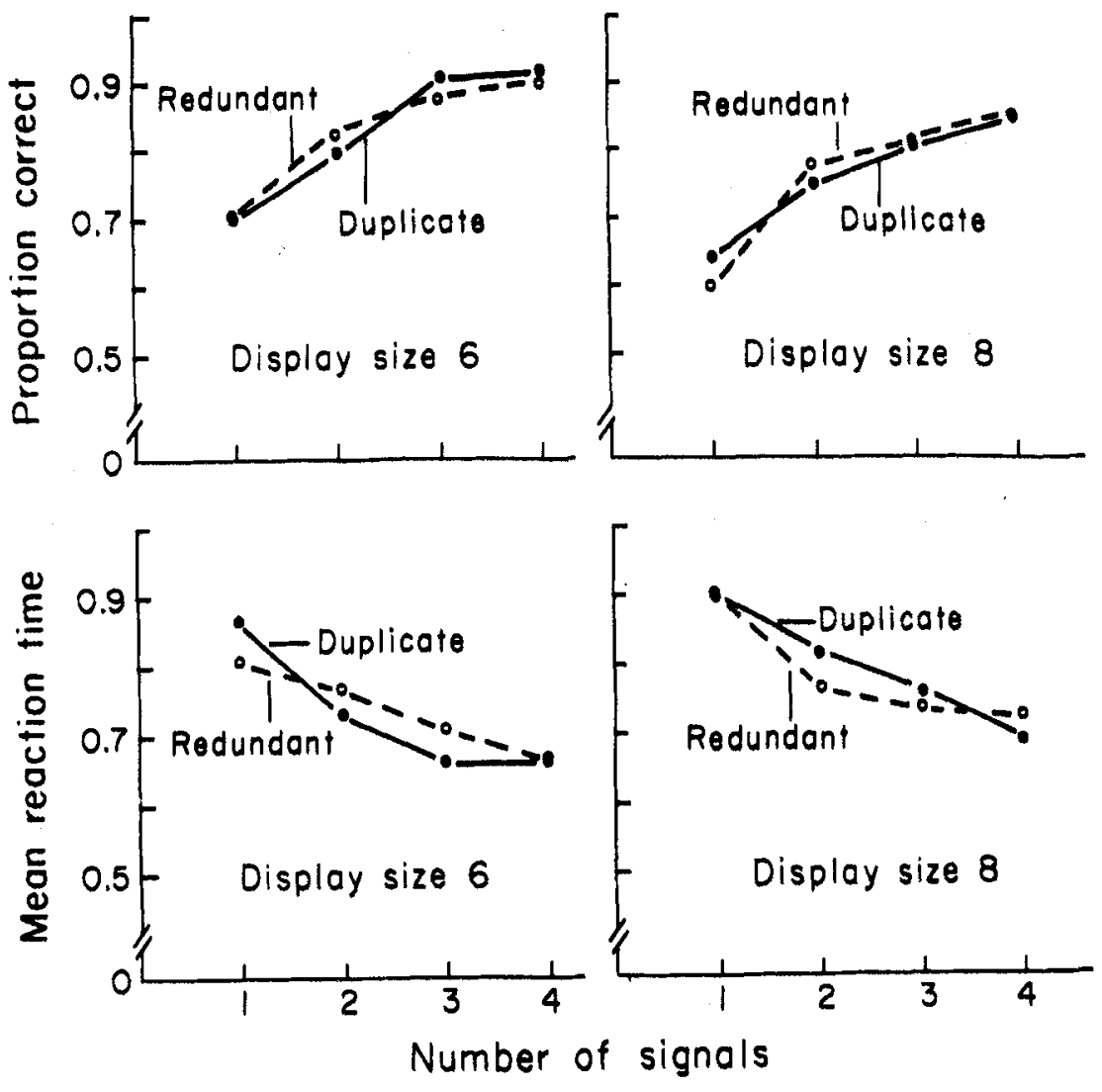

(1972) are somewhat reduced under the conditions of the present study. But even with signal-noise confusability strictly controlled, we find that increasing the heterogeneity of noise letters within a display significantly reduces both accuracy and speed of detection responses.

If detection were an all-or-none process, that is, if $S$ either identified the signal or guessed at random on each trial, our results on noise redundancy could not readily be interpreted at the decision level. However, the models of Estes (1972), Gardner (1973), and Shiffrin and Geisler (1973) all assume that upon a brief exposure of a letter, the input may activate a subset of the feature detectors which are required to identify the letter. If on a trial when a signal $S_{1}$ occurred, the $S$ obtained only an incompiete feature representation, he might still be able to decide correctly between $S_{1}$ and the alternative signal, $S_{2}$ if there were no noise letters present. But if the input from the display contained also a degraded representation of a noise letter which was highly confusable with $S_{2}$, a decision would have to be made by comparing the two inputs with the representations of the two possible signals in memory. This decision would take time, thus increasing the reaction time on the trial, and might eventuate in an error.

On the assumption that degraded inputs occur at least as frequently on heterogeneous as on homogeneous noise trials, the process of memory comparison and decision envisaged by the three models accounts nicely accuracy of detection. Further, our analysis of errors arising from various specific combinations of signal and noise letters appears to confirm the assumed process in considerable detail.

With respect to reaction times, the situation is a bit different. The models of Estes (1972) and Shiffrin and Geisler (1973) both assume that shortest reaction times will occur on trials when input from the signal letter activates its full feature set (yielding what I have termed "primary detections") and longer times on trials when the signal input is degraded and additional memory comparisons are required. But the model of Shiffrin and Geisler, as presently formulated, includes no mechanism that would entail any systematic relation between the proportion of primary detection trials and redundancy of noise elements, and the same appears to be true of

In the case of the interactive channels model, however, increasing heterogeneity of noise elements inputs from signals were masked by inhibitory interactions, thus giving rise to degraded representations (see Appendix 2). This effect of noise heterogeneity would be opposed by an increase in the multiplicity of signals present on a trial, and consequently the model implies the interaction of signal and noise redundancy observed in our reaction time data.

Another current model, the "all-at-once-scan" for the observed effect of noise heterogeneity upon Gardner's model. would lead to an increasing proportion of trials on which 
(AAOS) model proposed by McLaughlin et al (1972), implies an effect of redundancy at the perceptual level, but does so via a mechanism quite different from that of the interactive channels model.

The AAOS model is based on the conception of parallel processing of common features embodied in Selfridge's pandemonium model (Selfridge, 1959), postulating that information is extracted from the letters of the display by a serial scanning process, as assumed by Estes and Taylor (1966), but that once a given element has been sampled all other identical letters in the display are processed simultaneously before scanning proceeds to a different letter.

Predictions from the AAOS model yielded good agreement with observed detection rates for two degrees of redundancy of noise background in the study of McLaughlin et al (1972). However, their experiments did not test implications of the model with regard to effects of display size. Equation 3 of their paper gives the following expression for a measure of detection performance corrected for guessing:

$$
K_{D}=1+(D-1)\left(1-s_{1}\right),
$$

where $\mathrm{D}$ denotes display size and $s_{1}$ is the scan rate parameter. The theoretical quantity $\mathrm{K}_{\mathrm{D}}$ is estimated from data by the statistic $[\mathrm{P}(\mathrm{C})-\mathrm{P}(\mathrm{E})] \mathrm{D}$, that is, the difference between the proportion of correct detections and the proportion of errors multiplied by display size, and under some assumptions may be taken to represent the expected number of letters processed from the display. The reasoning leading to this equation can readily be extended to the case of multiple redundant signal elements, and in general takes the form

$$
\begin{aligned}
K_{D, m} & =m+(D-m)\left(1-s_{1}\right) \\
& =m s_{1}+\left(1-s_{1}\right) D,
\end{aligned}
$$

where $m$ denotes the number of instances of the signal element present on a trial. It is immediately apparent from this last expression that a plot of $K_{D, m}$ vs display size is predicted to yield a straight line with a slope that is independent of the number of redundant signals. In contrast, the interactive channels model implies an increase in slope with an increase in number of redundant signals within the range of this study (Appendix 2).

Estimates of $K_{D, m}$ computed from the observed response proportions of the present experiment decrease with display size, increase with number of signal elements, and are greater for the heterogeneous than for the homogeneous noise background. The pattern of significance levels is, of course, the same as for the correct response proportions. The main purpose in computing these estimates was to test the prediction from the AAOS scan model that, for the homogeneous background condition, the function relating $K_{D, m}$ to display size would have the same slope regardless of the number of signal elements. Considering only the cases of one and two signal elements in order to avoid ceiling effects, we obtain $K_{D, m}$ estimates of 2.6 and 4.0 for one signal element at Display Sizes 4 and 8, respectively, and 3.1 and 5.9 for two signal elements. The differences between the estimates for Display Sizes 4 and 8 are 1.4 and 2.8 in the cases of one and two signals, respectively, each value having an estimated standard error of .22 . Clearly, the prediction of constant slope was not confirmed.

Theoretical values of these same differences computed for the interactive channels model (see Appendix 2 are 1.8 and 2.7 , neither of which differs significantly from the observed value. Thus we seem to have some support for a model which assumes that the detrimental effects of additional different noise letters in a display are due in part to impaired perception of the signal letter.

The results of Experiment II suggest that the effects of signal redundancy may be localized almost entirely at the decision level. The effect of adding an additional signal which conveys the same information as the signals already present in a display appears to be independent of the physical similarity of the signal elements to each other. Evidently, additional redundant signals simply provide additional chances that the input from at least one will escape the masking effects of the noise background and lead to a correct detection response. It should be emphasized, however, that one should expect the result reported here to hold only under conditions such as those of the present experiment, in which many potential confounding variables have been meticulously controlled.

The issue of independent vs interactive input channels to feature detectors cannot be resolved by any one experiment. And, in fact, it is not even clear that we should be looking for a decision in the sense of finding one type of model right and the other wrong. It does seem that accumulating evidence (considering, for example, the findings of Eriksen \& Rohrbaugh, 1970; Estes \& Wolford, 1971; Townsend, Taylor, \& Brown, 1971; and the present study) implies that the input of information from tachistoscopic displays is limited by inhibitory effects of noise characters upon the perception of signal letters. This conclusion applies, however, only to displays in which relative letter spacing approximates that of ordinary printed matter. It is quite possible, and in fact is an assumption of the interactive channels model, that lateral inhibitory effects decrease with distance between letters. Consequently, it is to be expected that at sufficiently wide separations of the letters of a display, interactive effects would become negligible and performance might conform closely to that predicted from independent channels models.

\section{APPENDIX 1}

\section{EFFECTS OF NOISE REDUNDANCY AT THE DECISION LEVEL}

In the "independent channels" models of Gardner 
(1973) and Shiffrin and Geisler (1973), it is assumed that each character in a display activates an independent representation in memory. These representations may be degraded as a consequence of suboptimal exposure conditions. Adding noise characters to a display complicates the S's decision problem in that degraded noise inputs may be mistaken for signals and give rise to incorrect detection responses. Similar considerations would apply in the writer's formulation (Estes, 1972) following the stage of interactions at the perceptual level.

It is easy to show that these effects of noise elements at the decision level will be greater in a heterogeneous than in a homogeneous noise background, on the assumption that different noise letters vary in their probabilities of confusion with signal letters.

To illustrate the reasoning involved, consider an example of a simplified situation involving a set of just two noise characters, $\mathrm{N}_{1}$ and $\mathrm{N}_{2}$, and a miniexperiment in which each trial constitutes presentation of a signal element plus either two instances of $N_{1}$ or two instances of $\mathrm{N}_{2}$ (a homogeneous noise condition), or one instance each of $\mathrm{N}_{1}$ and $\mathrm{N}_{2}$ (a heterogeneous noise condition). Suppose that the signal letter would be detected with probability $p$ if presented alone. We will denote the interfering effects of the noise letters $\mathrm{N}_{1}$ and $\mathrm{N}_{2}$ by the "masking probabilities" $a$ and $b$, respectively, and assume that the signal is detected with probability $\mathrm{p}(1-\mathrm{a})^{2}, \mathrm{p}(1-\mathrm{b})^{2}$, or $\mathrm{p}(1-\mathrm{a})(1-\mathrm{b})$ when it is presented together with two instances of $\mathrm{N}_{1}$, two instances of $\mathrm{N}_{2}$, or one instance each of $\mathrm{N}_{1}$ and $\mathrm{N}_{2}$. Now, over a series of trials run under a homogeneous noise condition, the average probability of detection will be

$$
\frac{p}{2}\left[(1-a)^{2}+(1-b)^{2}\right]
$$

and under a heterogeneous noise condition $\mathrm{p}(1-\mathrm{a})(1-\mathrm{b})$. Subtracting the second expression from the first yields

$$
p\left[\frac{(1-a)^{2}+(1-b)^{2}}{2}-(1-a)(1-b)\right]=\frac{p}{2}(a-b)^{2},
$$

which is always positive if $a \neq b$, implying better detection in the homogeneous condition. But if $a=b$, the difference is zero, implying equal probabilities of detection in the homogeneous and heterogeneous backgrounds. This result holds, of course, only for experiments in which the frequencies of occurrence of the different noise letters on heterogeneous and homogeneous trials are balanced as in the example.

\section{APPENDIX 2}

\section{EFFECTS OF NOISE REDUNDANCY AT THE PERCEPTUAL LEVEL}

In the interactive channels model, variation in redundancy of noise elements affects target detection by modifying the opportunities for inhibitory interactions among input channels to feature detectors. The following representation of the model is considerably simplified for illustrative purposes, but preserves the essential relationships relevant to the present type of experiment. We shall assume that on the average $k$ additional feature detectors are activated by each different noise letter added to a display and that each feature detector activated by a noise letter has some probability, q, of reducing the perceptibility of the signal letter by a factor, a.

Case 1. One signal letter in a heterogeneous noise background. Denoting the probability of identification of the signal by $\mathbf{P}_{\mathrm{D}, 1}$, we have

$$
P_{D, 1}=p \alpha^{k(D-1)},
$$

where $\mathrm{D}$ denotes display size and $\mathrm{p}$ the probability of identification of the signal in the absence of noise elements, and $\alpha=1-q+q a$.

Case 2. Two signal letters in a heterogeneous noise background. Neglecting any inhibitory interactions of the two signal letters with each other,

$$
P_{D, 2}=2 p \alpha^{k(D-2)}-p^{2} \alpha^{2 k(D-2)} .
$$

To obtain Eq. ii, we reduce the exponent in Eq. $\mathrm{i}$ by unity, since there are only $D-2$ noise elements, add the probabilities of identification of the two signals separately, and subtract their product.

More generally, if there are $\mathrm{m}$ redundant signal letters in a display of Size D,

$$
\begin{aligned}
P_{D, m} & =m R_{D, m}-\left(\begin{array}{c}
m \\
2
\end{array}\right) R_{D, m}{ }^{2} \cdots \pm R_{D, m}^{m} \\
& =\sum_{i=1}^{m}(-1)^{i-1}\left(\begin{array}{c}
m \\
i
\end{array}\right) R_{D, m}{ }^{i},
\end{aligned}
$$

where $R_{D, m}=p \alpha^{k}(D-m)$.

Case 3. One signal letter in a homogeneous noise background. Since the features of noise elements exert their effects independently, we can first consider some one feature which is included in each of the identical noise letters. With probability $(1-q)^{D-1}$, this feature fails to be activated by the display; with probability $1-(1-q)^{\mathbf{D}-1}$, the feature is activated and reduces the perceptibility of the signal by a factor a. The same argument applies in the case of each of the $k$ noise features, and consequently probability of identification of the signal is given by

$$
P_{D, 1}=p\left\{(1-q)^{D-1}+\left[1-(1-q)^{D-1}\right] a\right\} k
$$

Term-by-term comparison of the expansions of Expressions $i$ and iv shows immediately that, as would be expected, the two are equal for $D=1$ and $D=2$, but for $D>2, P_{D, 1}$ is greater in the homogeneous background.

Case 4. Two signal letters in a homogeneous background. Letting $\mathbf{R}_{\mathbf{D}, \mathrm{m}}=\mathrm{p}\left\{(1-\mathrm{q})^{\mathbf{D}-\mathrm{m}}+\right.$ $\left.\left[1-(1-q)^{D-m}\right] a\right\} k$,

$$
P_{D, 2}=2 R_{D, 2}-R_{D, 2}{ }^{2}
$$


and, in general, $\mathbf{P}_{\mathbf{D}, \mathbf{m}}$ is given by an expression of the same form as Eq. iii.

To apply these results to the problem of interpreting the interaction of display size and number of redundant signals in determination of the quantity $K_{D, m}=D_{D, m}$ in the homogeneous background condition of Experiment I, we need only substitute appropriate numerical values in Eqs. iv and v. It can readily be shown that, in general, expressions for $K_{D, m}$ as a function of $D$ approach straight lines as $\mathrm{D}$ becomes large, but the slope for the case of two signals ( $2 \mathrm{pa}^{k}-\mathrm{p}^{2} \mathrm{a}^{2 k}$ ) is higher than that for one signal ( $\left.\mathrm{pa}^{\mathrm{k}}\right)$. Thus, the observed interaction is predicted by the interactive channels model. For the particular parameter values $\mathrm{p}=\mathrm{k}=1$ and $\mathrm{q}=\mathrm{a}=.5$, we obtain values of 2.2 and 4.0 for $K_{D, m}$ at display sizes of 4 and 8 , respectively, with one signal letter, and 3.4 and 6.1 with two signal letters. The corresponding values of $\mathrm{K}_{\mathrm{D}, \mathrm{m}}$ computed from the data are $2.6,4.0,3.1$, and 5.9 , in the same order.

\section{REFERENCES}

Eriksen, C. W., \& Rohrbaugh, J. W. Some factors determining efficiency of selective attention. American Journal of Psychology, 1970, 83, 330-342.

Estes, W. K. Interactions of signal and background variables in visual processing. Perception \& Psychophysics, 1972, 12, 278-286.
Estes, W. K., \& Taylor, H. A. Visual detection in relation to display size and redundancy of critical elements. Perception \& Psychophysics, 1966, 1, 369-373.

Estes, W. K., \& Wolford, G. L. Effects of spaces on report from tachistoscopically presented letter strings. Psychonomic Science, 1971, 25, 77-80.

Gardner, G. T. Evidence for independent parallel channels in tachistoscopic perception. Cognitive Psychology, 1973, 4 , 130-155.

Kinchla, R. A. Detecting target elements in multielement arrays: A confusability model. Perception \& Psychophysics, 1974, 15, 149-158.

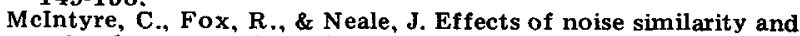
redundancy on the information processed from brief visual displays. Perception \& Psychophysics, 1970, 7, 328-332.

McLaughlin, J. P., Masterson, F. A., \& Herrmann, D. J. Pattern redundancy and detection in very short-term memory. Perception \& Psychophysics, 1972, 12, 205-208.

Selfridge, $O$. G. Pandemonium: A paradigm for learning. In $T$ he mechanization of thought processes. London: Her Majesty's Stationary Office, 1959 .

Shiffrin, R. M., \& Geisler, W. S. Visual recognition in a theory of information processing. In $R$. L. Solso (Ed.), Contemporary issues in cognitae psychology: The Loyola symposium. New York: Winston, 1973. Pp. 53-101.

Townsend, J. T., Taylor, S. G., \& Brown, D. R. Lateral masking for letters with unlimited viewing time. Perception \& Psychophysics, 1971, 10, 375-378.

Wolford, G. L., Wessel, D. L., \& Estes, W. K. Further evidence concerning scanning and sampling assumptions of visual detection models. Perception \& Psychophysics, 1968, 3, 439-444.

(Received for publication October 24, 1973; revision accepted February 25, 1974.) 